Revue

Revue de l'histoire des religions

de Ihistoire des religions

$1 \mid 2018$

Varia

Joseph F. BYRNES, Priests of the French Revolution.

Saints and Renegades in a New Political Era

University Park, The Pennsylvania State University Press, 2014

Rémy Hême de Lacotte

\title{
CpenEdition
}

Journals

Édition électronique

URL : http://journals.openedition.org/rhr/8884

DOI : $10.4000 /$ rhr.8884

ISSN : 2105-2573

Éditeur

Armand Colin

Édition imprimée

Date de publication : 1 mars 2018

Pagination : 183-186

ISBN : 978-2-200-93166-7

ISSN : 0035-1423

Référence électronique

Rémy Hême de Lacotte, « Joseph F. Byrnes, Priests of the French Revolution. Saints and Renegades in a New Political Era », Revue de l'histoire des religions [En ligne], 1 | 2018, mis en ligne le 20 mars 2018, consulté le 15 janvier 2021. URL : http://journals.openedition.org/rhr/8884 ; DOI : https://doi.org/ $10.4000 /$ rhr.8884

Ce document a été généré automatiquement le 15 janvier 2021.

Tous droits réservés 


\section{Joseph F. BYRNES, Priests of the French Revolution. Saints and Renegades in a New Political Era}

University Park, The Pennsylvania State University Press, 2014

Rémy Hême de Lacotte

\section{RÉFÉRENCE}

Joseph F. BYRnES, Priests of the French Revolution. Saints and Renegades in a New Political Era, University Park, The Pennsylvania State University Press, 2014, 22,5 cm, XXVI-314 p., \$79,95, ISBN 978-0-271-063776-5.

1 Annoncé de divers côtés comme la première synthèse universitaire sur le clergé constitutionnel, l'ouvrage de Joseph Byrnes se révèle assurément plus complexe, par son objet comme par sa forme. Le premier se déduit du titre, à dessein très général : même si tous les ecclésiastiques réunis dans ces pages ont prêté le serment requis par le décret du 27 novembre 1790, c'est moins cette adhésion qui retient ici l'attention de l'auteur que ce qu'elle exprime de leur participation au processus révolutionnaire, leur véritable dénominateur commun. Quant à la forme, plutôt que le tableau d'ensemble auquel on s'attendrait a priori, elle consiste en une succession de portraits individuels, disposés suivant la trame chronologique, chacune des figures évoquées - pour certaines d'entre elles à plusieurs reprises - étant associée à un moment de l'histoire de ce clergé " révolutionnaire ». Ce parti pris se comprend mieux si l'on sait que le présent travail n'est qu'une pièce d'un chantier collectif, dont les jalons ont été posés en juin 2012 à Lyon par le colloque «Gouverner une Église en Révolution. Histoires et mémoires de l'épiscopat constitutionnel français » (actes parus en 2017). Il devrait aboutir à la publication d'un dictionnaire biographique de l'épiscopat constitutionnel, destiné à se substituer à celui - plutôt orienté - du chanoine Pisani (1907), ainsi qu'à la mise en 
ligne d'un répertoire du clergé assermenté. Loin d'une somme définitive, l'ensemble s'apparente donc à une contribution d'étape.

2 Sur un plan factuel, le propos conforte nos connaissances bien plus qu'il ne les bouleverse, à l'image du découpage adopté, des plus classiques : des prodromes de la Révolution à la création de la nouvelle structure ecclésiastique ; de la division du clergé et des fidèles face au serment aux campagnes de déchristianisation de l'an II ; de la reconstitution d'une Église (désormais qualifiée de "gallicane ", pour marquer sa différence avec la première) après Thermidor à la normalisation concordataire sous le Consulat. Les principaux épisodes de l'histoire religieuse de la Révolution ne sont volontairement retracés que dans leurs grandes lignes : quatre pages pour le Comité ecclésiastique de l'Assemblée constituante, à peine plus pour la question du serment (bien défrichée par Timothy Tackett). Une chronologie détaillée rappelle il est vrai, en tête de chaque partie, les repères essentiels. La troisième partie est un peu plus prolixe. Joseph Byrnes y insiste avec raison, dans la lignée des travaux de Bernard Plongeron et de Rodney J. Dean et données précises à l'appui, sur le relèvement opéré par les anciens constitutionnels à compter de 1795 autour de Grégoire et des évêques réunis, malgré une présence encore aléatoire à l'échelle du territoire national en 1801. On peine cependant à prendre exactement la mesure de cette entreprise, faute d'une comparaison avec l'activité pastorale déployée au même moment par les réfractaires, qui aurait éclairé le lecteur sur le rapport de forces entre les deux clergés à la veille du concordat.

3 L'intérêt principal de l'ouvrage réside, on l'aura compris, dans sa riche galerie de portraits, là où les histoires générales se contentent de quelques noms (que domine celui de Grégoire). Ce n'est pas que les figures du clergé constitutionnel n'aient jamais été étudiées : nombre d'entre elles, au contraire, ont fait l'objet de biographies, mais celles-ci sont le produit d'une historiographie datée, mêlée, à l'occasion, d'intentions apologétiques. La thèse publiée de Caroline Chopelin-Blanc, De l'apologétique à l'Église constitutionnelle: Adrien Lamourette (1742-1794), Paris, 2009 (à laquelle il est largement fait référence dans le passage consacré à ce dernier prélat), ou celle en cours d'Emmanuel Lacam sur l'évêque de Versailles Clément montrent la voie d'un renouvellement bienvenu dans le champ universitaire - dont témoigne encore la toute récente étude de l'ecclésiologie de La Font de Savine par Francesco Dei, La Chiesa senza leggi : religione e potere secondo un vescovo della Rivoluzione francese (1791-1794), Brescia, 2014. Dix-sept personnalités sont ici plus particulièrement mises en lumière, toutes membres d'assemblées révolutionnaires et appartenant majoritairement (pour douze d'entre elles) à l'épiscopat. Le panel est contrasté, les uns s'illustrant avant tout dans la sphère ecclésiale, à l'image de l'évêque d'Ille-et-Vilaine Le Coz, quand d'autres doivent leur notoriété à leur militantisme révolutionnaire, tel l'abbé Sieyès ou le terroriste Joseph Le Bon. L'auteur fait par ailleurs une place à des acteurs plus modestes, tels ces prêtres livrant leurs lettres de prêtrise ou sollicitant au contraire du légat pontifical Caprara leur réconciliation, dont il reproduit quelques témoignages. Un des fils conducteurs de l'ouvrage est une réflexion sur l'articulation entre identité sacerdotale et engagement révolutionnaire. Les cas de figure sont là aussi variés, qui vont de la fidélité à leur état à sa brutale négation, en passant par toutes sortes d'alliages. Les analyses de J. Byrnes font ressortir la singularité et l'imprévisibilité de ces itinéraires, par-delà le caractère parfois convenu du discours, notamment chez les abdicataires, soucieux d'authentifier leur républicanisme par une critique en règle de leur condition passée. Si le 
détachement d'un Sieyès vis-à-vis du dogme est précoce, ses confrères semblent avoir évolué plus tardivement, s'acquittant scrupuleusement de leurs tâches pastorales et cultivant une authentique vie spirituelle. Leurs sources d'inspiration tiennent alors tout à la fois de l'idéal d'utilité sociale du clergé, entretenu par les Lumières, et d'une lecture messianique des événements révolutionnaires comme entreprise de libération du nouveau peuple de Dieu. La résistance des réfractaires à la nouvelle Église accompagne un premier mouvement de radicalisation et la remise en cause des modèles sacerdotaux traditionnels, en même temps que se font entendre des revendications audacieuses (abolition du célibat ecclésiastique, emploi du vernaculaire dans le culte - ce dernier point est encore débattu sous le Directoire). La tendance s'accentue encore avec l'échec de la monarchie constitutionnelle et l'adhésion à la déchéance, puis (pour une partie) à la condamnation de Louis XVI, longtemps épargné, voire célébré (voir p. 130 l'élogieux sermon du futur leader enragé Jacques Roux). L'époque de la Convention consacre, sur fond de persécution du clergé catholique, la dissolution de l'identité constitutionnelle entre des options spirituelles et politiques divergentes (les plus engagés dans la violence révolutionnaire se répartissant euxmêmes entre des obédiences antagonistes). La perpétuation de ce clergé, au sortir de la Terreur, passait dès lors nécessairement par une refondation.

4 Une autre évolution sensible à la lecture est précisément celle des fondements intellectuels et doctrinaux de l'expérience constitutionnelle. Sur l'ecclésiologie, on repère des constantes : la revendication du retour à la discipline primitive de l'Église et l'inscription dans la tradition gallicane, nonobstant quelques inflexions ou controverses internes (entre défenseurs de l'autorité épiscopale et tenants des idées presbytériennes par exemple). Les connexions avec le jansénisme sont évoquées, mais sans que la question soit vraiment creusée. Il en va différemment de ce que l'on pourrait qualifier de «rapport au monde». Les animateurs et théoriciens de l'Église constitutionnelle première formule, à l'exemple de Lamourette, promeuvent un catholicisme rénové, en dialogue - critique - avec les philosophes des Lumières voire les francs-maçons, véritable « religion nationale » (titre d'un manifeste du futur évêque du Calvados Fauchet). Sans abandonner cette prétention englobante, les artisans de la renaissance d'une Église "gallicane» en 1795 adoptent une position davantage défensive, étayée par le principe de la liberté des cultes. Le serment à la constitution civile du clergé fait encore figure de moment fondateur, mais perd de sa valeur intrinsèque. Le concordat de 1801 engage ce qu'il reste de constitutionnels dans un combat mémoriel pour la défense de leurs choix passés. Qu'un certain nombre de prêtres rétractés se retranchent pour justifier leur serment derrière la soumission due aux lois et le nécessaire maintien du culte montre cependant le recul enregistré en une décennie par rapport au dessein originel.

5 Au terme de ce parcours, on retiendra Priests of the French Revolution comme un ouvrage singulier: partiel, sinon partial (quelques passages reflètent, à tout le moins, une subjectivité très sincèrement assumée), parfois diffus, mais aussi très incarné, faisant une large place aux documents d'époque et rempli de vues suggestives, dont le lecteur tirera profit, dans l'attente des publications ultérieures qu'il annonce. 


\section{AUTEURS}

\section{RÉMY HÊME DE LACOTTE}

Centre d'histoire du XIX siècle (Paris 1-Paris IV). 